\title{
Production of fast ion and nuclei bunches of solid densities from nanolayers by ultrarelativistic laser pulses
}

\begin{abstract}
H. K. Avetissian, * A. K. Avetissian, G. F. Mkrtchian, and Kh. V. Sedrakian
Centre of Strong Fields Physics, Yerevan State University, 1 A. Manukian, Yerevan 0025, Armenia (Received 29 April 2011; revised manuscript received 10 June 2011; published 19 October 2011)

A method for generation of quasimonoenergetic, low emittance fast ion/nuclei bunches of solid densities from nanotargets by two counterpropagating laser pulses of ultrarelativistic intensities is proposed, based on the threshold phenomenon of particles reflection due to induced nonlinear Compton scattering. Particularly, a setup is considered which provides generation of ion bunches with parameters that are required in hadron therapy.
\end{abstract}

DOI: 10.1103/PhysRevSTAB.14.101301

PACS numbers: 41.75.Jv, 37.10.Vz, 52.38.Kd, 52.65.Rr

\section{INTRODUCTION}

Acceleration of ions with superstrong laser beams and their interaction with the matter at extreme conditions in ultrashort space-time scales have attracted broad interest over the last few years conditioned by a number of important applications, such as generation and probing of highenergy-density matter [1], inertial confinement fusion [2], isotope production [3], hadron therapy [4], etc. Most of these mechanisms are based on the indirect processes, when ions are accelerated by the space-charge fields induced in the laser-target interaction process. There are several regimes of ions acceleration depending on the laser intensity: target normal sheath acceleration regime [5], Coulomb explosion regime [6], radiation pressure dominant regime [7], and the shock wave acceleration mechanism [8]. Typically, in the target normal sheath acceleration regime, the accelerating gradient reaches $10^{12} \mathrm{~V} / \mathrm{m}$ but, due to short interaction range $\sim 1 \mu \mathrm{m}$, it accelerates the ions to tens of $\mathrm{MeV}$ energies. The energy distribution of accelerated ions obtained by the laserplasma interaction is usually exponential with almost $100 \%$ energy spread up to a cutoff energy. Only within the last few years, the production of monoenergetic ion beams from targets using a special technique became possible [9]. In these experiments up to $60 \mathrm{MeV}$ protons were observed. In the radiation pressure dominant regime, with circularly polarized laser pulses when heating of electrons is reduced, it is expected to achieve $\mathrm{GeV} /$ nucleon energy range at the ultrarelativistic laser intensities [10].

The use of the mentioned indirect processes for ion acceleration, because of the lack of directly accelerating laser fields of required intensities, has some undesirable

\footnotetext{
*avetissian@ysu.am
}

Published by the American Physical Society under the terms of the Creative Commons Attribution 3.0 License. Further distribution of this work must maintain attribution to the author(s) and the published article's title, journal citation, and DOI. factors, such as uncontrollable beam parameters, etc. The state-of-the-art laser systems are capable of generating electromagnetic pulses with intensities exceeding on several orders the threshold of relativism for electrons. For heavier particles, laser intensities, at which, e.g., an ion with atomic mass number $\mathcal{A}$ and charge number $\mathcal{Z}$ becomes relativistic, is defined by the condition $\Xi \geq 1$, where

$$
\Xi=\frac{Z_{e E \lambda}}{\mathcal{A} m_{u} c^{2}}
$$

is the relativistic dimensionless parameter of a waveparticle interaction ( $e$ is the elementary charge, $c$ is the light speed in vacuum, and $m_{u}$ is the atomic mass unit $m_{u} \simeq 1.66 \times 10^{-24} \mathrm{~g}$ ) and represents the work of the field with electric strength $E$ on a wavelength $\lambda(\lambda=\lambda / 2 \pi)$ in the units of particle rest energy. Laser intensities, at which relativistic effects become important for an ion ( $\Xi=1$-threshold value) can be estimated as

$$
I_{r}=\Xi^{2} \mathcal{A}^{2} Z^{-2} \times 4.55 \times 10^{24} \mathrm{~W} \mathrm{~cm}^{-2}[\lambda / \mu \mathrm{m}]^{-2} .
$$

The availability of such intensities now is in the scope of the extreme light infrastructure (ELI) project [11] and the mechanisms for direct laser acceleration of heavy particles are of interest. The spectrum of direct acceleration mechanisms of nuclei/ions by a single laser pulse is very restricted, since one should use the laser beams focused to subwavelength waist radii, or use subcycle laser pulses the acceleration rate for which is relatively small in comparison with the processes in the presence of additional resonances. Direct acceleration of ions by petawatt laser beams focused to subwavelength waist radii has been explored with linearly and radially polarized lasers [12], where ion energies cover the domain of application in hadron therapy [4]. However, the realization of this scheme with radially polarized laser pulses of required ultrarelativistic intensities is problematic. In the paper [13], it has been considered a combination of the laser-plasma mechanism as a proton source with the postacceleration employing single 
and crossed pulsed laser beams focused to spot radii on the order of the laser wavelength.

From the point of view of low energy spreads and emittances of accelerated ions/nuclei bunches, it is reasonable to consider coherent processes of laser-particle interaction with the additional resonances. Among those the induced Compton process is practically more effective. Thus, in this process a critical value of intensity exists [14] above which the nonlinear threshold phenomenon of particles reflection or capture occurs from the slowed interference wave moving potential barrier or well formed by two counterpropagating laser pulses [15]. This phenomenon opens a principally new way for particles acceleration on the short distances - even shorter than a laser wavelength. For acceleration in the capture regime, the phase velocity of the slowed interference wave must be changed along the propagation direction (for gaseous media with variable refractive index and for counterpropagating laser beams in vacuum with the frequency variation) $[15,16]$. Note that the acceleration of protons $\sim 10 \mathrm{MeV}$ by this way in the capture regime due to chirped pulse has been considered in [17].

In this paper we propose a method for generation of solid density, monoenergetic, and low emittance ion/nuclei bunches of ultrashort durations. Accordingly, we will study laser acceleration of ions/nuclei in the induced Compton process with strongly nonplane ultrashort pulses, taking into account the specific feature of the critical field for generation of fast ion beams with low energy spreads and emittances. For realization of the latter we propose to focus two counterpropagating laser beams of different frequencies and ultrarelativistic intensities onto a nanoscale-solidplasma target with $Z / \mathcal{A}=1 ; 1 / 2$ (for protons and nuclei, respectively). In particular, we consider the specific range of energies that is of great interest to hadron therapy [4] with quasimonoenergetic-monochromatic ion beams.

The organization of the paper is as follows. In Sec. II the problem of laser acceleration is formulated analytically on the base of classical relativistic equations of motion for a charged particle in the fields of two counterpropagating plane laser pulses of different frequencies, and the conditions of the above-threshold interaction regime are stated corresponding to the reflection phenomenon. In Sec. III we present numerical calculations for the ionized hydrogen ${ }^{1} \mathrm{H}^{+}$and fully ionized carbon ${ }^{12} \mathrm{C}^{6+}$ targets based on the particle-in-cell (PIC) simulation code. Finally, conclusions are given in Sec. IV.

\section{NONLINEAR DYNAMICS OF AN ION IN THE FIELD OF COUNTERPROPAGATING LASER PULSES IN VACUUM}

At first we consider the classical dynamics of an ion in vacuum at the interaction with two counterpropagating plane waves of carrier frequencies $\omega_{1}, \omega_{2}$ (let $\omega_{1}>\omega_{2}$ ), wave numbers $\mathbf{k}_{1}=\left\{\omega_{1} / c, 0,0\right\}, \mathbf{k}_{2}=\left\{-\omega_{2} / c, 0,0\right\}$, and slowly varying electric field amplitudes $E_{1}\left(\tau_{1}\right), E_{2}\left(\tau_{2}\right)$ $\left(\tau_{1}=t-x / c, \tau_{2}=t+x / c\right)$. Both waves are assumed to be linearly polarized along the $O Y$ direction:

$$
\mathbf{E}_{1,2}(x, t)=\left\{0, E_{1,2}\left(\tau_{1,2}\right) \cos \omega_{1,2} \tau_{1,2}, 0\right\} .
$$

Dynamics of an ion in the resulting electric $\mathbf{E}=\mathbf{E}_{1}+\mathbf{E}_{2}$ and magnetic $\mathbf{H}=\hat{\mathbf{x}} \times\left(\mathbf{E}_{1}-\mathbf{E}_{2}\right)$ fields is governed by the equations:

$$
\frac{d \boldsymbol{\Pi}}{d t}=\frac{e Z}{\mathcal{A} m_{u} c}\left(\mathbf{E}+\frac{\boldsymbol{\Pi} \times \mathbf{H}}{\gamma}\right), \quad \frac{d \gamma}{d t}=\frac{e Z}{\mathcal{A} m_{u} c} \frac{\boldsymbol{\Pi} \cdot \mathbf{E}}{\gamma} .
$$

Here we have introduced normalized momentum $\boldsymbol{\Pi}=$ $\mathbf{p} /\left(\mathcal{A} m_{u} c\right)$ and energy $\gamma=\sqrt{1+\Pi^{2}}$ (Lorentz factor). When the particle initial transversal momentum is zero, and the waves are turned on/off adiabatically at $t \rightarrow \mp \infty$, from Eq. (2) for transversal momentum one can obtain (coordinate $z$ is cyclic, so $\Pi_{z}=$ const $\equiv 0$ )

$$
\Pi_{y}\left(\Xi_{1}, \Xi_{2}\right)=\Xi_{1}\left(\tau_{1}\right) \sin \omega_{1} \tau_{1}+\Xi_{2}\left(\tau_{2}\right) \sin \omega_{2} \tau_{2} .
$$

With the help of Eq. (3), one can obtain equations for longitudinal momentum and energy, which include four nonlinear interaction terms: two of them are proportional to $\Xi_{1,2}^{2}\left(\tau_{1,2}\right) \sin 2 \omega_{1,2} \tau_{1,2}$ and describe interaction with separate waves that cannot provide real energy change for the ion. The term proportional to $\Xi_{1}\left(\tau_{1}\right) \Xi_{2}\left(\tau_{2}\right) \times$ $\sin \left(\omega_{1} \tau_{1}+\omega_{2} \tau_{2}\right)$ describes interaction with the fast interference wave making no contribution to real energy exchange too. This term is responsible for particleantiparticle pair production from the Dirac vacuum [18]. The resonant interaction of the ion for acceleration is governed by the slowed interference wave $\Xi_{1}\left(\tau_{1}\right) \Xi_{2}\left(\tau_{2}\right) \times$ $\sin \tilde{\omega}\left(t-x / c \beta_{\mathrm{ph}}\right)$, where $\tilde{\omega}=\omega_{1}-\omega_{2}$ and $\beta_{\mathrm{ph}}=$ $\tilde{\omega} /\left(\omega_{1}+\omega_{2}\right)<1$ are the frequency and normalized phase velocity $\left(\mathrm{v}_{\mathrm{ph}} / c\right)$ of the slowed wave. Hence, keeping only this resonant term, one can obtain the following integral of motion in average:

$$
\gamma-\beta_{\mathrm{ph}} \Pi_{x} \simeq \gamma_{0}-\beta_{\mathrm{ph}} \Pi_{0 x} .
$$

From Eqs. (3) and (4) and dispersion relation $\gamma=\sqrt{1+\boldsymbol{\Pi}^{2}}$, one can see that for the certain values of $\Xi_{1,2}$ (which we call critical), the following relation for an ion average transversal momentum in the field may be satisfied:

$$
\sqrt{\overline{\Pi_{y}^{2}\left(\Xi_{1}, \Xi_{2}\right)}}>\gamma_{0} \frac{\left|\beta_{\mathrm{ph}}-\beta_{0 x}\right|}{\sqrt{1-\beta_{\mathrm{ph}}^{2}}}
$$

$\left(\beta_{0 x}=\mathrm{v}_{0 x} / c\right.$, where $\mathrm{v}_{0 x}$ is the initial longitudinal velocity of the ion) at which the slowed interference wave becomes a potential barrier causing ion reflection from this moving wave barrier. This process is the classical induced nonlinear Compton effect which is of coherent nature like the Cherenkov process with a slowed wave in a dielectric medium electromagnetic radiation in the form of plane 
traveling wave. Because of the coherent feature (Compton resonance between the wave frequencies with Doppler shifts) of this process, the nonlinear interaction dynamics with a charged particle has threshold character by the wave intensities or by the slowed interference wave intensity, which we called critical $\left(\Xi_{\mathrm{cr}}\right)$. The mentioned phenomenon takes place only if the intensity parameter $\Xi_{\text {eff }}$ of the resulting interference wave (slowed wave in vacuum due to Compton resonance) is larger than this critical one, at which the effective traveling wave becomes a potential barrier for an external particle or a potential well for a particle initially situated in the wave. The physical meaning of critical intensity is that at this value the particle longitudinal velocity in the field become equal to phase velocity of the slowed effective wave, irrespective of its initial value before the interaction:

$$
\left.\mathrm{v}_{\|}\left(t, \Xi_{1,2}\right)\right|_{x=x(t)}=\mathrm{v}_{\mathrm{ph}} \equiv c \frac{\omega_{1}-\omega_{2}}{\omega_{1}+\omega_{2}},
$$

which is the classical condition (without quantum recoil) of induced Compton resonance with counterpropagating waves. Since $\mathrm{v}_{\|}\left(t, \Xi_{1,2}\right)$ is determined by the expression which depended on the intensities of strong waves (see [15] for induced Cherenkov, Compton, and undulator processes) and Compton resonance becomes accessible in the field due to the waves' intensities effect (initially the particle is off resonance), then this resonance is principally nonlinear and of threshold nature because of the process coherent character. Hence, in this critical point nonlinear Compton resonance occurs in the result of which the particle velocity becomes a greater induced inverse Compton effect or smaller induced direct Compton effect (depending on its initial longitudinal velocity: $\mathrm{v}_{0 x}<\mathrm{v}_{\mathrm{ph}}$ or $\mathrm{v}_{0 x}>\mathrm{v}_{\mathrm{ph}}$ ) than the wave phase velocity and it leaves the wave. So, at $\Xi_{\text {eff }}>\Xi_{\text {cr }}$ the particle reflection from the wave barrier or capture in the wave well occurs (double reflection from each wall of the slowed wave wells). After the interaction $\left(\Xi_{1,2}=0\right)$ for the reflected ion final energy we have [15]

$$
\gamma_{f} \simeq \gamma_{0}+2 \gamma_{0} \beta_{\mathrm{ph}} \frac{\beta_{\mathrm{ph}}-\beta_{0 x}}{1-\beta_{\mathrm{ph}}^{2}}
$$

Formula (7) shows that, because of the impact character of the particle-barrier interaction, the energy of the reflected particle depends neither on the waves' field magnitudes nor the interaction length (only the threshold condition: $\Xi_{\text {eff }}>\Xi_{\text {cr }}$ must be satisfied). Namely, this feature of reflection phenomenon is used here for generation of monoenergetic and low emittance ions/nuclei bunches of ultrashort durations.

\section{GENERATION OF ION/NUCLEI BUNCHES FROM NANOTARGETS: PIC SIMULATIONS}

Then for actual strongly nonplane and supershort ultrarelativistic laser pulses of certain configurations, the problem is solved with the help of PIC simulations. Here we report on the results of the 2D3V PIC simulations of counterpropagating wave interaction with nanolayers. We use the code XOOPIC, which is a relativistic code based on the PIC method [19]. Then we consider two types of ion targets: ionized hydrogen ${ }^{1} \mathrm{H}^{+}$and fully ionized carbon ${ }^{12} \mathrm{C}^{6+}$. The simulation box size is $40 \lambda_{1} \times 20 \lambda_{1}$ in the $x y$ plane. The number of cells is $4000 \times 200$. The total number of macroparticles is about $4 \times 10^{4}$ for the hydrogen target and $2.4 \times 10^{5}$ for the carbon target. The target is assumed to be fully ionized. This is justified since the intensity for full ionization of carbon is about $10^{19} \mathrm{~W} / \mathrm{cm}^{2}$, while we use in simulations intensities at least 5 orders of magnitude larger. So, the target will become fully ionized well before the arrival of the pulses' peaks. Electrons and ions are assumed to be cold in the target, $T_{e}=T_{i}=0$.

The laser pulses have profiles $\sin ^{2}\left(\pi t / \mathcal{T}_{1,2}\right)$ with pulse durations $\mathcal{T}_{1}=5 \lambda_{1} / c, \mathcal{T}_{2}=3 \lambda_{2} / c$ and Gaussian transverse profiles: $\exp \left(-r^{2} / w_{1,2}^{2}\right)$, with the waists $w_{1}=6 \lambda_{1}$ and $w_{2}=3 \lambda_{2}$. The carrier-envelope phase of the lasers is set to zero, so that the electric fields' maximums are at the pulses' centers. The first laser $\left(\omega_{1}\right)$ is introduced at the left boundary and propagates along the $x$ axis from left to right, focused at the target layer. The second laser $\left(\omega_{2}\right)$ is introduced at the right boundary and is also focused at the target layer. We consider the case $\lambda_{1}=\lambda_{2} / 2$ when $\lambda_{2}=800 \mathrm{~nm}$ which for the phase velocity of the slowed wave gives $\beta_{\text {ph }}=1 / 3$, and according to Eq. (7) for reflected particle normalized kinetic energy gives $\gamma_{f}-1 \approx 0.25$. In numerical simulations, for laser intensities it is assumed $\Xi_{1}=0.6, \Xi_{2}=0.3$, at which the intensity of the effective slowed wave is above the critical point.

Before PIC simulations, we have cleared up the role of initial conditions. For this goal, we have integrated Eq. (2) numerically for the various initial position $x_{0}$ of an ion in rest: $\mathbf{v}_{0}=0$. The integration of the relativistic equations of motion in the given field of counterpropagating pulses, initially centered at the distances $\pm 25 \lambda_{1}$, has been made with the fourth-order adaptive Runge-Kutta method. The result of integration, final energy versus the initial position $x_{0} \in\left[-\lambda_{1} ; \lambda_{1}\right]$, is displayed in Fig. 1. In case of waves adiabatic turn on/off, the energy of the reflected particles should be independent on the initial position $x_{0}$. However, for short laser pulses depending on the initial position of an ion, reflection will take place with various velocities, since before overlapping of laser pulses (for the formation of a slowed interference wave in vacuum responsible for reflection phenomenon) due to short rise time of laser profiles, ions will acquire various velocities depending on its initial positions. As is seen from Fig. 1, there are plateaus where 


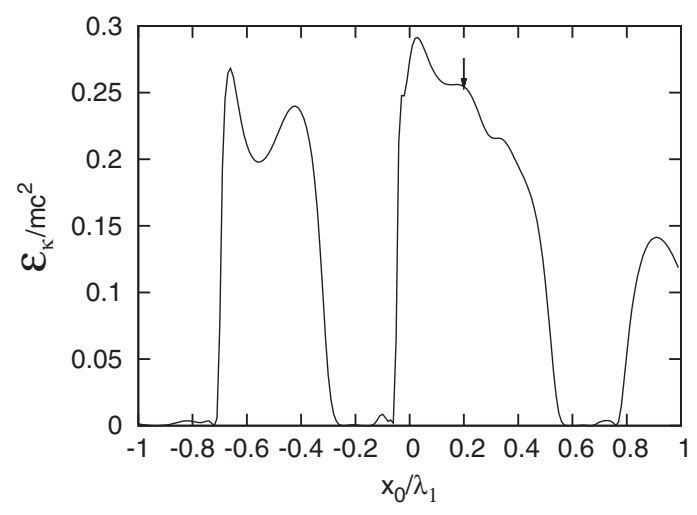

FIG. 1. The final scaled kinetic energy versus the initial position of the ion $x_{0}$ in units of wavelength $\lambda_{1}$. The arrow shows position of the target layer.

one would have reflection independent on the ion initial position. Hence, the arrow in Fig. 1 (which corresponds to relative position of the target layer) shows the position of such a plateau. Figure 1 shows that the used model with the phenomenon of particle reflection, which in principle is valid for waves adiabatic turn on/off, is also accurate for ultrashort pulses in predicting the final energies of reflected particles: $\gamma_{f}-1 \approx 0.25$, in accordance with Eq. (7). Thus, taking into account this result, for PIC simulations the target layer is placed at the distance of $15.2 \lambda_{1}$ from the left boundary, and the first laser is introduced with time delay to start the pulse: $t_{\mathrm{del}}=10 \lambda_{1} / c$ for economy of computational time. The simulation box size in the propagation direction have been taken to be $40 \lambda_{1}$. Without time delay for the box size, one should take $50 \lambda_{1}$ selecting the position of a nanolayer at the distance $25.2 \lambda_{1}$ from the left boundary (with the additional $10 \lambda_{1}$ one would describe only the free propagation of the first laser beam, which is eliminated choosing the appropriate time delay).

In Fig. 2, the ion layer blown out from a thin ${ }^{1} \mathrm{H}^{+}$target of thickness $8 \mathrm{~nm}$ by counterpropagating waves is shown for various instants. Here $n_{e}=n_{c}$, where as a measure of density we take critical plasma density $n_{c}=1.74 \times$

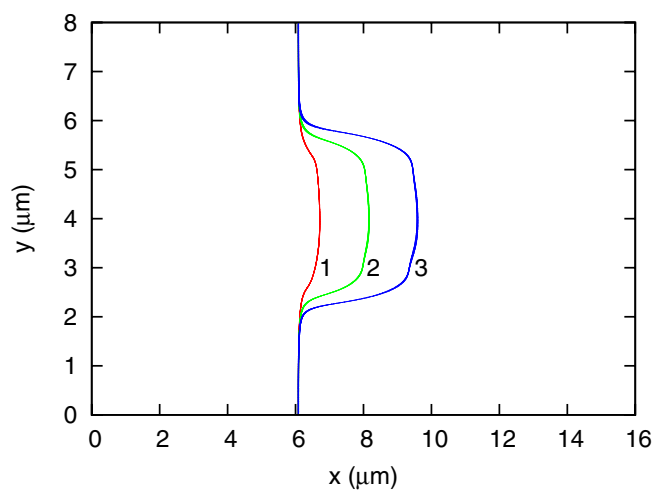

FIG. 2. Ion layer blown out from a thin foil by counterpropagating waves. The $(x-y)$ phase space for protons at instants (1) $15 T_{2}$, (2) $18 T_{2}$, and (3) $21 T_{2}$.
$10^{21} \mathrm{~cm}^{-3}$ calculated for $8 \mathrm{~nm}$ laser. Note that electrons are escaped from the target before the arrival of the pulses' peaks. After the time period $21 T_{2}$, the ions are already free. The final kinetic energy $\mathcal{E}_{k}$ and angular $\phi=$ $\tan ^{-1}\left(\Pi_{x} / \Pi_{y}\right)$ distributions of accelerated protons versus the transversal position are shown in Figs. 3 and 4, respectively. The number density of protons is displayed in Fig. 5.

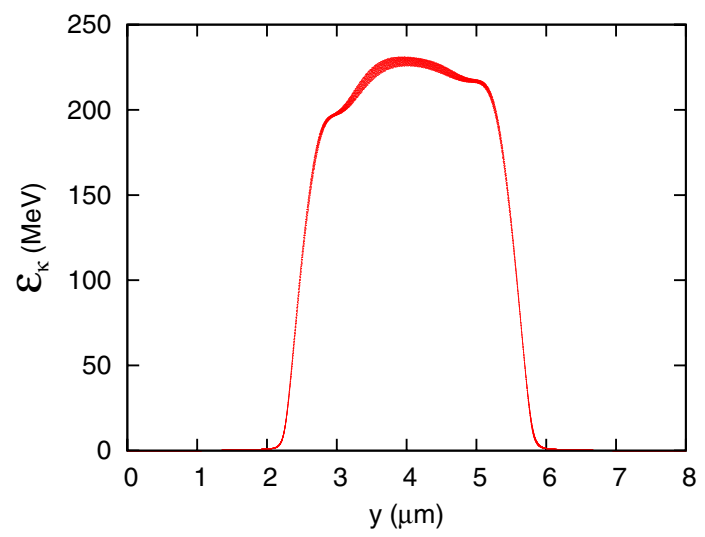

FIG. 3. The final kinetic energy distribution (at instant $21 T_{2}$ ) of accelerated protons versus the transversal position $y$.

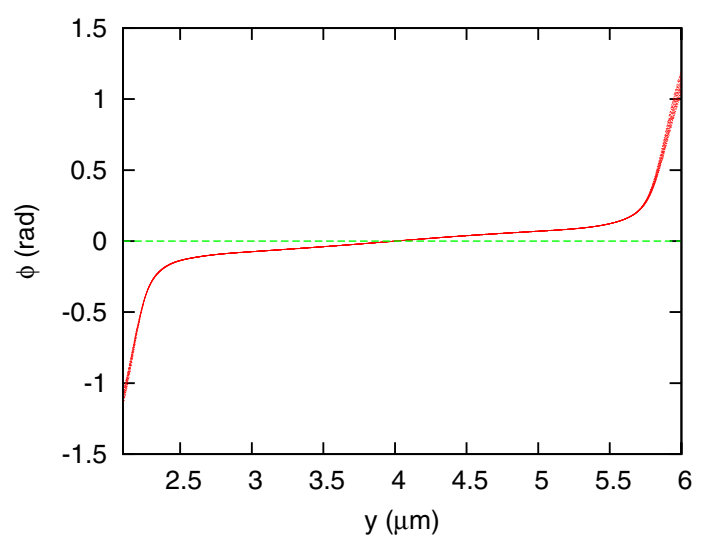

FIG. 4. The angular distribution of accelerated protons versus the transversal position $y$.

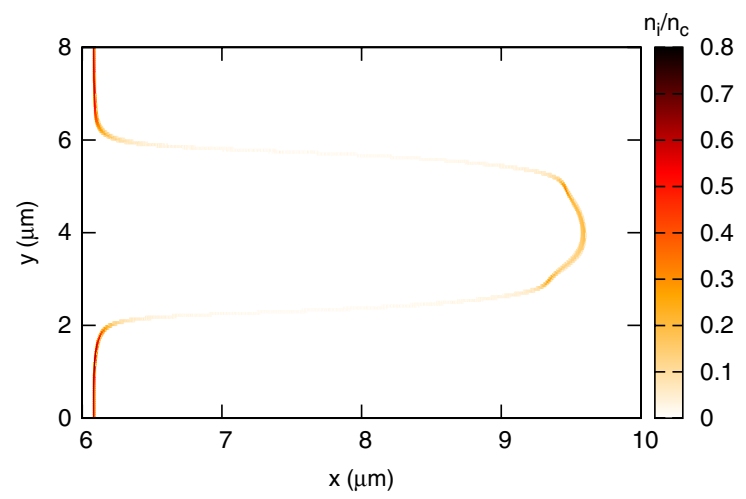

FIG. 5. Density distribution of protons at instant $21 T_{2}$. 


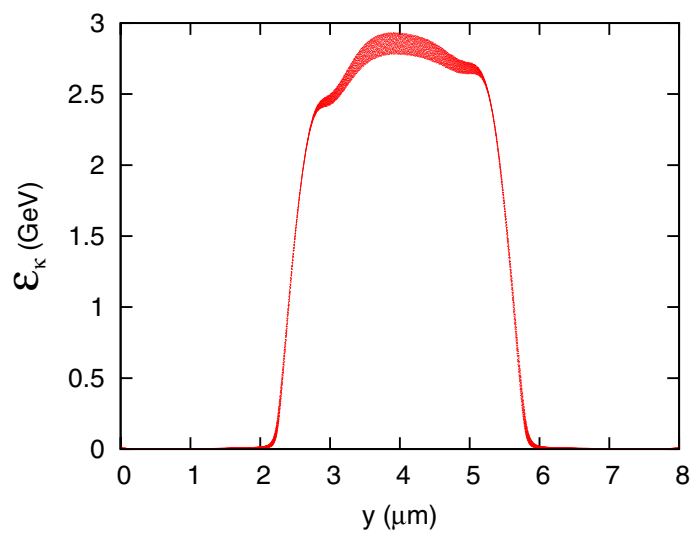

FIG. 6. The final kinetic energy distribution of carbon ions versus the transversal position $y$.

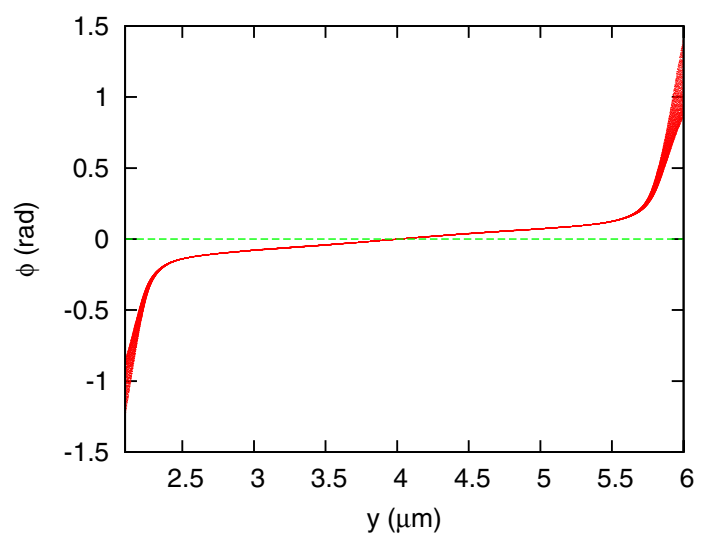

FIG. 7. The angular distribution of accelerated carbon ions versus the transversal position $y$.

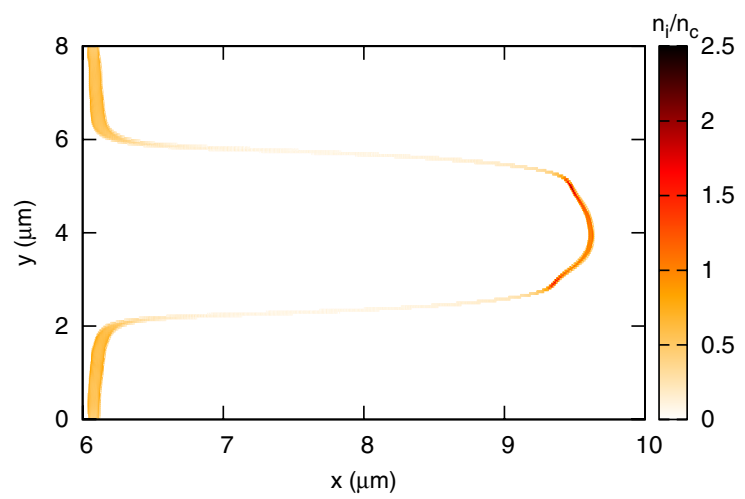

FIG. 8. Density distribution for carbon ions at instant $21 T_{2}$.

The results for carbon foil of density $n_{e}=80 n_{c}$ and $4 \mathrm{~nm}$ thickness are shown in Figs. 6-8. As is seen from these figures, although charge to mass ratio is different for ${ }^{12} \mathrm{C}^{6+}$, the interaction picture for both cases of protons and carbon ions looks qualitatively the same. Because of the larger space-charge fields for carbon ions, the energy and angular spreads are slightly larger compared with the protons.
As the employed laser pulses are of nonplane configuration, here there is, in principle, an acceleration effect of particles even in the field of a single pulse. However, the latter is negligibly small (in accordance with the wellknown Lawson-Woodward theorem [20]) where, for assessing purposes, we have also made simulations with a single pulse interaction introduced at the left boundary. In Fig. 9 the ion layer is illustrated after the interaction (at the instant $21 T_{2}$ ) with a single pulse having intensity corresponding to maximal value employed in this work: $\Xi_{1}=0.6$. For this setup the final kinetic energy $\left(E_{k}\right)$ distribution versus the transversal position is shown in Fig. 10. As Figs. 9 and 10 show, the residual acceleration because of nonplane character of a focused laser beam is negligibly small compared with the acceleration due to reflection phenomenon.

To estimate emittances of the ions bunch, we will consider the effective area limited to the central zone between $3.5 \mu \mathrm{m}<y<4.5 \mu \mathrm{m}$. Estimates for transverse $\left(\epsilon_{t}\right)$ and longitudinal $\left(\epsilon_{l}\right)$ emittances of the ions bunch show that for protons $\epsilon_{t}<0.1 \pi \mathrm{mm}$ mrad and $\epsilon_{l}<10^{-8} \mathrm{eV} \mathrm{s}$, while for

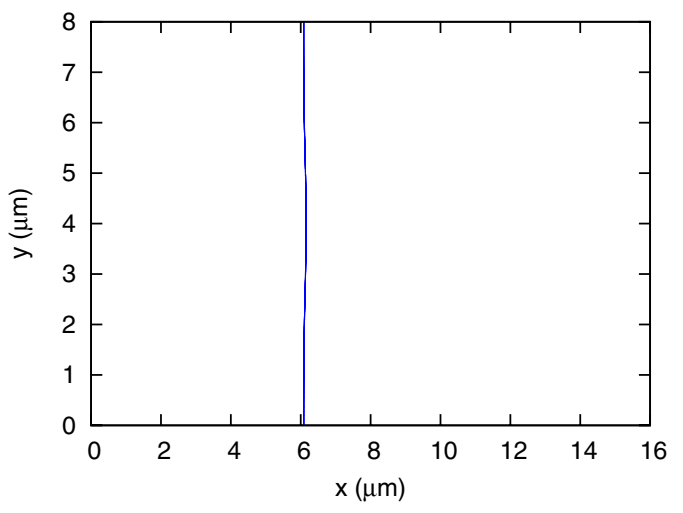

FIG. 9. Proton layer after the interaction with single laser pulse $\left(\Xi_{1}=0.6\right)$ introduced at the left boundary.

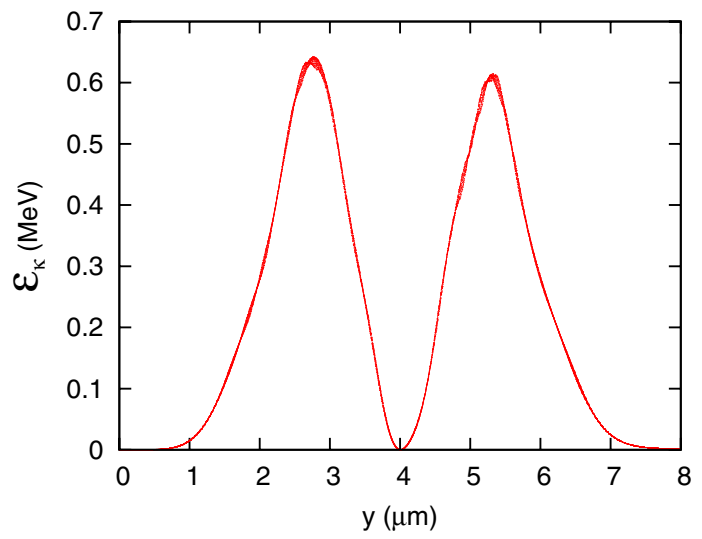

FIG. 10. The final kinetic energy distribution (at instant $21 T_{2}$ ) of protons versus the transversal position $y$ for the interaction with single laser pulse ( $\left.\Xi_{1}=0.6\right)$ introduced at the left boundary. 
carbon ions $\epsilon_{t}<0.1 \pi \mathrm{mmmrad}$ and $\epsilon_{l}<10^{-7} \mathrm{eV} \mathrm{s}$. These results are several orders of magnitude smaller than their counterparts in conventional ion accelerators. The corresponding energy spreads are $\delta \mathcal{E} / \mathcal{E} \sim 10^{-2}$.

\section{CONCLUSION}

We have proposed a nonlinear mechanism for generation of quasimonoenergetic and low emittance heavy particle bunches of solid densities from nanotargets by counterpropagating laser pulses of ultrarelativistic intensities and different frequencies. The considered method of generation of fast ion/nuclei bunches is based on the nonlinear threshold phenomenon of particles reflection from the slowed interference wave-pulse formed in the induced Compton process. Because of the impact feature of the particle-wave-barrier interaction at the extremely short lengths, the mentioned phenomenon allows one to generate high brightness particle bunches of solid densities from nonrelativistic to relativistic energies due to variation of the lasers' frequencies ratio in the wide range. In parallel to analytic treatment, the proposed mechanism has been demonstrated with PIC simulations. Particularly, a setup is considered which provides generation of ion bunches in the range of such energies and low spreads that may have application in hadron therapy.

\section{ACKNOWLEDGMENTS}

This work was supported by State Committee of Science (SCS) of the Republic of Armenia (RA).

[1] D. H. H. Hoffmann et al., Laser Part. Beams 23, 47 (2005).

[2] M. Roth et al., Phys. Rev. Lett. 86, 436 (2001); A. Macchi et al., Nucl. Fusion 43, 362 (2003); M. Temporal, J. J. Honrubia, and S. Atzeni, Phys. Plasmas 15, 052702 (2008); J. J. Honrubia et al., ibid. 16, 102701 (2009); N. Naumova et al., Phys. Rev. Lett. 102, 025002 (2009).

[3] S. Fritzler et al., Appl. Phys. Lett. 83, 3039 (2003); E. Lefebvre et al., J. Appl. Phys. 100, 113308 (2006).

[4] E. Fourkal et al., Med. Phys. 29, 2788 (2002); V. Malka et al., Med. Phys. 31, 1587 (2004); S. S. Bulanov et al., ibid. 35, 1770 (2008); I. Hofmann, J. Meyer-ter-Vehn, X. Yan, A. Orzhekhovskaya, and S. Yaramyshev, Phys. Rev. ST Accel. Beams 14, 031304 (2011).
[5] S. C. Wilks et al., Phys. Plasmas 8, 542 (2001); T.Z. Esirkepov et al., Phys. Rev. Lett. 89, 175003 (2002); H. B. Zhuo et al., ibid. 105, 065003 (2010).

[6] S. V. Bulanov et al., Phys. Lett. A 299, 240 (2002); E. Fourkal, I. Velchev, and C.-M. Ma, Phys. Rev. E 71, 036412 (2005); S. S. Bulanov et al., ibid. 78, 026412 (2008).

[7] T. Esirkepov, M. Borghesi, S. V. Bulanov, G. Mourou, and T. Tajima, Phys. Rev. Lett. 92, 175003 (2004); S. G. Rykovanov et al., New J. Phys. 10, 113005 (2008); A. A. Gonoskov, A. V. Korzhimanov, V.I. Eremin, A. V. Kim, and A. M. Sergeev, Phys. Rev. Lett. 102, 184801 (2009); S. V. Bulanov et al., ibid. 104, 135003 (2010).

[8] L. O. Silva et al., Phys. Rev. Lett. 92, 015002 (2004).

[9] S. Ter-Avetisyan et al., Phys. Rev. Lett. 96, 145006 (2006); B. M. Hegelich et al., Nature (London) 439, 441 (2006); H. Schwoerer et al., Nature (London) 439, 445 (2006).

[10] A. Macchi, F. Cattani, T. V. Liseykina, and F. Cornolti, Phys. Rev. Lett. 94, 165003 (2005); A. Macchi, S. Veghini, and F. Pegoraro, ibid. 103, 085003 (2009); X. Q. Yan, H. C. Wu, Z.M. Sheng, J.E. Chen, and J. Meyer-ter-Vehn, ibid. 103, 135001 (2009); A. Henig et al., ibid. 103, 245003 (2009).

[11] The extreme light infrastructure (ELI) project: http:// www.extreme-light-infrastructure.eu/eli-home.php.

[12] Y. I. Salamin, Z. Harman, and C. H. Keitel, Phys. Rev. Lett. 100, 155004 (2008).

[13] B. J. Galow, Z. Harman, and C. H. Keitel, Opt. Express 18, 25950 (2010).

[14] V. M. Haroutunian and H. K. Avetissian, Phys. Lett. 59A, 115 (1976).

[15] H. K. Avetissian, Relativistic Nonlinear Electrodynamics (Springer, New York, 2006).

[16] H. K. Avetissian, S. S. Israelyan, and Kh. V. Sedrakian, Phys. Rev. ST Accel. Beams 10, 071301 (2007); H. K. Avetissian and Kh. V. Sedrakian, ibid. 13, 101304 (2010); 13, 081301 (2010).

[17] F. Peano, J. Vieira, L. O. Silva, R. Mulas, and G. Coppa, New J. Phys. 10, 033028 (2008).

[18] H. K. Avetissian, A. K. Avetissian, G. F. Mkrtchian, and Kh. V. Sedrakian, Phys. Rev. E 66, 016502 (2002).

[19] J. P. Verboncoeur, A. B. Langdon, and N. T. Gladd, Comput. Phys. Commun. 87, 199 (1995).

[20] P. M. Woodward, J. Inst. Electr. Eng. 93, 1554 (1947); J. D. Lawson, IEEE Trans. Nucl. Sci. 26, 4217 (1979). 